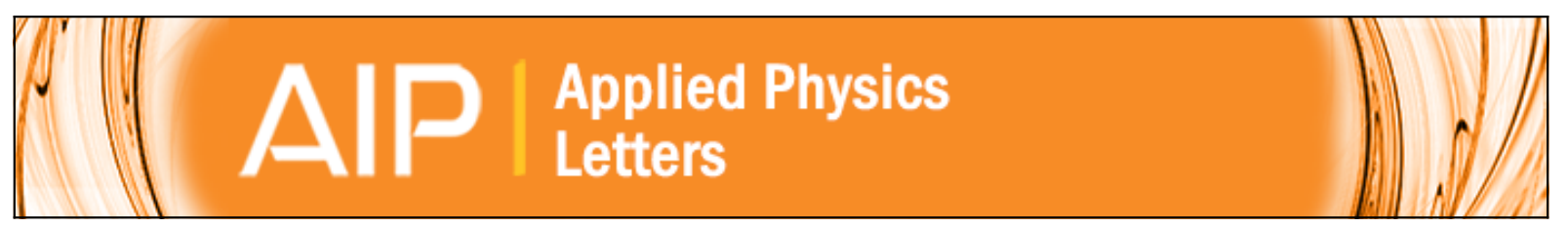

\title{
Direct detection of spontaneous polarization in wurtzite GaAs nanowires
}

Benedikt Bauer, Joachim Hubmann, Matthias Lohr, Elisabeth Reiger, Dominique Bougeard, and Josef Zweck

Citation: Applied Physics Letters 104, 211902 (2014); doi: 10.1063/1.4880209

View online: http://dx.doi.org/10.1063/1.4880209

View Table of Contents: http://scitation.aip.org/content/aip/journal/apl/104/21?ver=pdfcov

Published by the AIP Publishing

\section{Articles you may be interested in}

Quenching of the E 2 phonon line in the Raman spectra of wurtzite GaAs nanowires caused by the dielectric polarization contrast

Appl. Phys. Lett. 103, 043121 (2013); 10.1063/1.4817078

In-situ x-ray characterization of wurtzite formation in GaAs nanowires

Appl. Phys. Lett. 100, 093103 (2012); 10.1063/1.3688489

Growth of size and density controlled GaAs / In x Ga 1 x As / GaAs ( $x=0.10$ ) nanowires on anodic alumina membrane-assisted etching of nanopatterned GaAs

J. Vac. Sci. Technol. B 28, 1111 (2010); 10.1116/1.3498753

Type II heterostructures formed by zinc-blende inclusions in InP and GaAs wurtzite nanowires

Appl. Phys. Lett. 97, 041910 (2010); 10.1063/1.3473779

Facet structure of GaAs nanowires grown by molecular beam epitaxy

Appl. Phys. Lett. 91, 083106 (2007); 10.1063/1.2769401

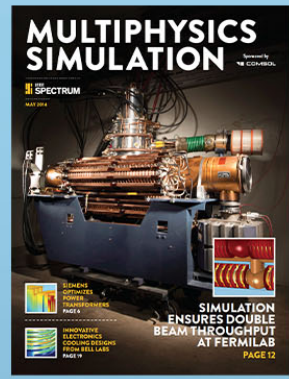

Free online magazine

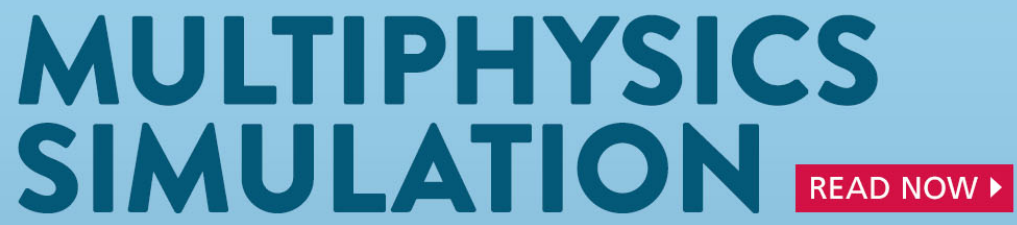




\title{
Direct detection of spontaneous polarization in wurtzite GaAs nanowires
}

\author{
Benedikt Bauer, ${ }^{\text {a) }}$ Joachim Hubmann, Matthias Lohr, Elisabeth Reiger, \\ Dominique Bougeard, and Josef Zweck ${ }^{\text {b) }}$ \\ Institut für Experimentelle und Angewandte Physik, Universität Regensburg, Germany
}

(Received 6 May 2014; accepted 14 May 2014; published online 27 May 2014)

\begin{abstract}
We demonstrate the direct detection of spontaneous polarization in the wurtzite crystal phase of gallium-arsenide (GaAs) nanowires. Using differential phase contrast microscopy (DPC) in a scanning transmission electron microscope, we map the differences in charge distribution between the zincblende and wurtzite crystal phases and use twin defects in the zinc-blende phase to quantify the polarization strength. The value of $2.7 \times 10^{-3} \mathrm{C} / \mathrm{m}^{2}$ found for the polarization strength matches well with theoretical predictions. (C) 2014 AIP Publishing LLC. [http://dx.doi.org/10.1063/1.4880209]
\end{abstract}

It is well known that due to their catalytic growth mode III-V-semiconductor nanowires (NWs) can grow in crystal structures that differ from the stable bulk crystal structurecircumstance that is often referred to as crystal structure polytypism. ${ }^{1-5}$ This allows to study the properties of crystal phases which are not or only rarely accessible in bulk or $2 \mathrm{D}$ materials. In our case, this is gallium-arsenide (GaAs) in the wurtzite (WZ) phase, which has mostly been subject to theoretical works ${ }^{6}$ so far, as there have been only very few publications about $\mathrm{WZ}$ in $\mathrm{GaAs}$ layers ${ }^{7}$ or in bulk crystals after a special pressure treatment. ${ }^{8}$

At first sight, the zinc-blende (ZB) and WZ crystal structures differ only in the stacking sequence, which is $A B C A B C$ and $A B A B A B$, respectively. Structurally this causes a lower symmetry of the WZ with only one instead of four equal symmetry axes compared to the ZB. On the atomic level, the orbitals of the $\mathrm{sp}^{3}$-hybridized atoms no longer form a symmetric tetrahedron but the bond in [0001] direction is shortened (in the non-Nitride case) or elongated (Nitride-based). In the non-Nitride III-V-materials, i.e., the Arsenides, Phosphides, and Selenides, whose bulk structure is ZB, this results in a narrower lattice spacing along the WZ [0001] direction $^{9}$ which corresponds to one of the four $\langle 111\rangle$ axes of the ZB structure. This leads to a non-uniform distribution of the orbitals around the core and therefore a displacement of the centers of positive and negative charges associated with "sheets" of alternating charge density along the [0001] axis.

This phenomenon which-among polarization phenomena of different origins-affects the electronic and optical properties of the material is called spontaneous polarization and is a well known issue in the (WZ structure) III-Nitrides. ${ }^{9,10}$ Especially at the interfaces of heterostructures polarization leads to accumulation of charges and therefore internal electric fields which are known to be the reason for the quantum-confined Stark effect (QCSE). ${ }^{11,12}$

In contrary, spontaneous polarization has not been of importance in other III-V-semiconductors yet as it does not occur in the stable ZB crystal phase. As WZ-GaAs is available now in NWs two publications addressed the problem of spontaneous polarization in such materials theoretically ${ }^{9}$ and

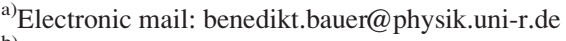

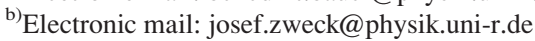

in its potential effect on the luminescence spectra. ${ }^{13}$ Both groups estimate the spontaneous polarization in WZ-GaAs to be one order of magnitude lower than in the III-Nitrides. In the following, we will present direct experimental evidence of the spontaneous polarization in WZ-GaAs as well as a quantification of the polarization strength based on the electric field between isolated twin stacking faults (SFs) via differential phase contrast microscopy (DPC).

DPC in a scanning transmission electron microscope (STEM) is a long established technique for the measurement of magnetic fields ${ }^{14}$ and has recently been demonstrated to be capable to map also internal electric fields and charge distributions on nanometer ${ }^{15}$ and even sub- $\AA$ length scales. ${ }^{16}$ The application of this technique on the measurement of internal electric fields has been discussed in detail in Ref. 15.

We use a FEI Tecnai F30 transmission electron microscope (TEM) where the standard annular dark field detector has been replaced by a custom made DPC detector that consists of a ring-shaped detector area divided into four segments that can be read out separately. To denote their position, the segments are named " 3 ," "6," "9," and " 12 " corresponding to the numbers seen on a clock face. Prior to image acquisition, the electron beam is aligned on the detector such that the electron diffraction disc illuminates only a small inner rim of the detector ring and that all four quadrants are illuminated equally. While the beam is scanned over the sample the signals from the four segments are read simultaneously for every beam position. If there is a deflecting field within the sample, the detector segments are no longer illuminated equally but the intensity is reduced on some segments and increased on the opposing ones. The setup has been calibrated to determine the electric field within the sample from the measured beam deflection.

By calculating the intensity differences $I_{3-9}$ and $I_{12-6}$ of opposing quadrants, the signal caused by the deflecting field is separated from that of non-directional scattering processes. The resulting difference images represent the beam deflection along two perpendicular coordinate axes $\hat{x}_{1}$ and $\hat{x}_{2}$ defined by the connecting axes of opposing detector segments. The intensity of these difference images is proportional to $E \times t$, where $E$ is the electric field perpendicular to the electron trajectory and $t$ is the thickness of the irradiated sample position. ${ }^{17}$ For geometries with the electric field 
perpendicular to the electron trajectory, it is possible to calculate the electric field within the sample by

$$
\vec{E}(x, y)=\alpha \times \frac{I_{3-9}(x, y) \times \hat{x}_{1}+I_{12-6}(x, y) \times \hat{x}_{2}}{t(x, y) \times I_{\text {Sum }}(x, y)} .
$$

Here, $\alpha$ is a calibration factor that describes the relation between the electric field and the resulting difference signal, $t$ is the local sample thickness the electron beam has traversed, and $I_{\text {Sum }}$ is the integrated intensity over all four detector segments which is needed to eliminate the influence of variable local absorption conditions.

As polarization acts like layers of positive and negative charge, the effect on the beam electrons can be treated in analogy to a plate capacitor filled with a dielectric. Therefore, one can calculate the spontaneous polarization $P_{\mathrm{sp}}$ from the electric field by $P_{\mathrm{sp}}=\varepsilon \varepsilon_{0}|\vec{E}|$. Furthermore, we calculate the divergence $\vec{\nabla} \cdot \vec{E}(x, y) \sim \rho^{\prime}(x, y)$ which is proportional to the charge density $\rho$ that the beam electrons have experienced in average along their way through the specimen at a certain beam position $(x, y)$, hence we call these images "charge distribution maps."

The presented data was taken from a GaAs NW sample that was grown by MBE in pseudo-Ga catalyzed growth mode $^{18}$ on a GaAs (111)B substrate covered with a $0.5 \AA$ gold layer. At a temperature of $528^{\circ} \mathrm{C}, \mathrm{GaAs}$ NWs were grown with a growth rate of $2.8 \AA / \mathrm{s}$ and an $\mathrm{As}_{4}$ beam equivalent pressure of $3.2 \times 10^{-6}$ Torr.

For the TEM investigations, NWs were transferred to a carbon coated TEM grid by simply wiping the grid over the as-grown sample. For the recording of the presented data, a NW was oriented in a $\langle 11 \overline{2} 0\rangle_{\mathrm{Hex}}$ (corresponding to $\langle 1 \overline{1} 0\rangle_{\mathrm{Cub}}$ ) zone axis which ensures that the electron beam transverses the sample perpendicular to the growth direction and therefore perpendicular to the expected electric field vectors, meeting the requirements for Eq. (1).

Figure 1(a) shows the upper part of a typical NW from this sample. Those NWs typically show twinned ZB crystal

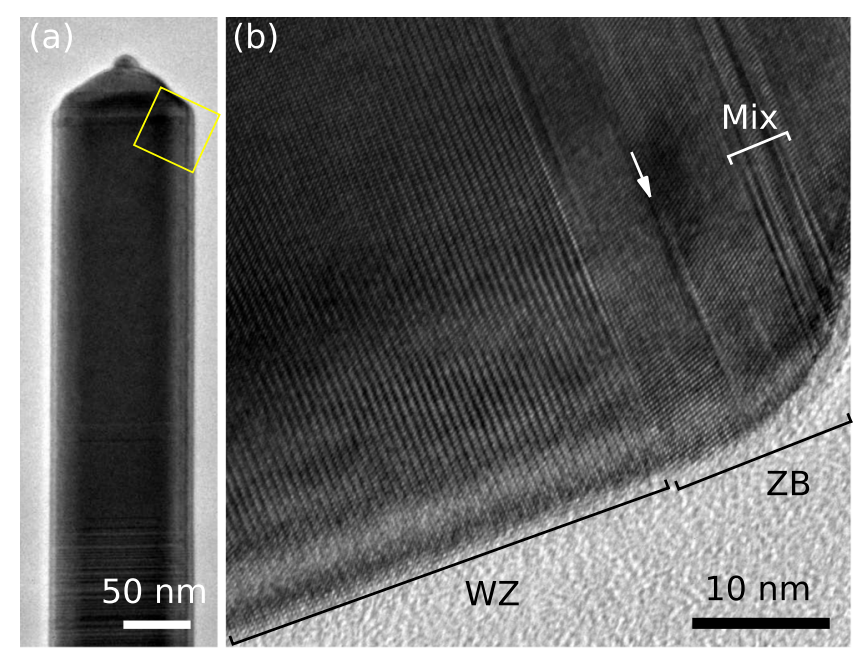

FIG. 1. TEM images of the investigated NW: (a) Overview of the NW tip. (b) High resolution TEM image of the square region in (a) depicting the WZ body, a ZB tip with two very closely related twin stacking faults (white arrow), and a section with alternating very thin $\mathrm{ZB}$ and $\mathrm{WZ}$ crystal structure segments ("Mix"). structure over the majority of their length $(\$ 5.5 \mu \mathrm{m})$, while the upper end can be characterized by a several $100 \mathrm{~nm}$ long transition region from $\mathrm{ZB}$ to $\mathrm{WZ}$ with a high stacking fault (SF) density and another $\approx 300 \mathrm{~nm}$ long segment of SF free WZ. The morphology of the whole upper part can be associated with the termination of the growth process, where the Ga supply is closed but an $\mathrm{As}_{4}$ atmosphere is retained until the substrate has cooled down. ${ }^{18}$ In Fig. 1(b), it can be clearly seen that the crystal structure switches back from WZ to $\mathrm{ZB}$ a few $\mathrm{nm}$ before the NW diameter reduces towards the droplet. The ZB region contains two closely related twin stacking faults (marked with a white arrow) and a mix region where the crystal structure changes ZB-WZ-ZB-WZ-ZB, each segment only some monolayers thick ("Mix").

In the charge distribution map in Fig. 2, taken from the same region as Fig. 1(b), electrically neutral regions show up as neutral gray, whereas charged areas appear brighter or darker. The superimposed curve is a line scan profile across the same image to allow for a better judgment of the signal amplitudes. The features identified in Fig. 1(b) can be found also in the charge distribution map. It can be clearly seen that the WZ in the left third of the image shows pronounced alternating positive and negative charges which leads to a stripe pattern of nearly constant amplitude, while the ZB region is neutral with only some noise. DPC thus delivers direct evidence for the existence of spontaneous polarization in the WZ phase of GaAs and its absence in the ZB phase.

However, in the line scan profile the WZ-ZB interface and the small ZB segment between the WZ segments of the "Mix" section (both marked with black arrows in Fig. 2) appear to be significantly more polarized than the WZ itself. Also their direct surroundings do not show a clear and consistent behavior. This can be explained by the image acquisition process: The electron spot has an Airy Function like intensity profile and the intensity of every image pixel is the result of the convolution of the electron beam profile with the probed potential landscape. As for the device used here the FWHM of the central maximum of the electron beam is about $1 \mathrm{~nm}$, which corresponds to about 1.5 lattice constants of WZ-GaAs along the [0001] axis, ${ }^{6,7}$ several positivenegative transitions are within the probed volume. In the pure WZ, this leads to an averaging effect and therefore to an underestimation of the charge density. In contrast, in regions where different crystal phases are in direct vicinity the image intensity is strongly influenced by the interplay

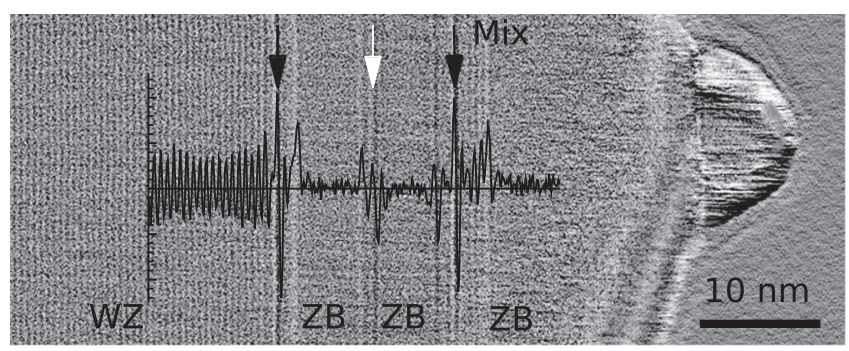

FIG. 2. Charge distribution map $\rho^{\prime}(x, y)$ at the NW tip across regions of WZ and $\mathrm{ZB}$ with overlaid intensity profile. The WZ structure and interfaces between different crystal phases (black and white arrows) show clear positive-negative transitions, whereas $\mathrm{ZB}$ areas appear in neutral, but noisy gray, i.e., they are uncharged. The white arrow and the "Mix" label correspond to the features as seen in Fig. 1(b). 


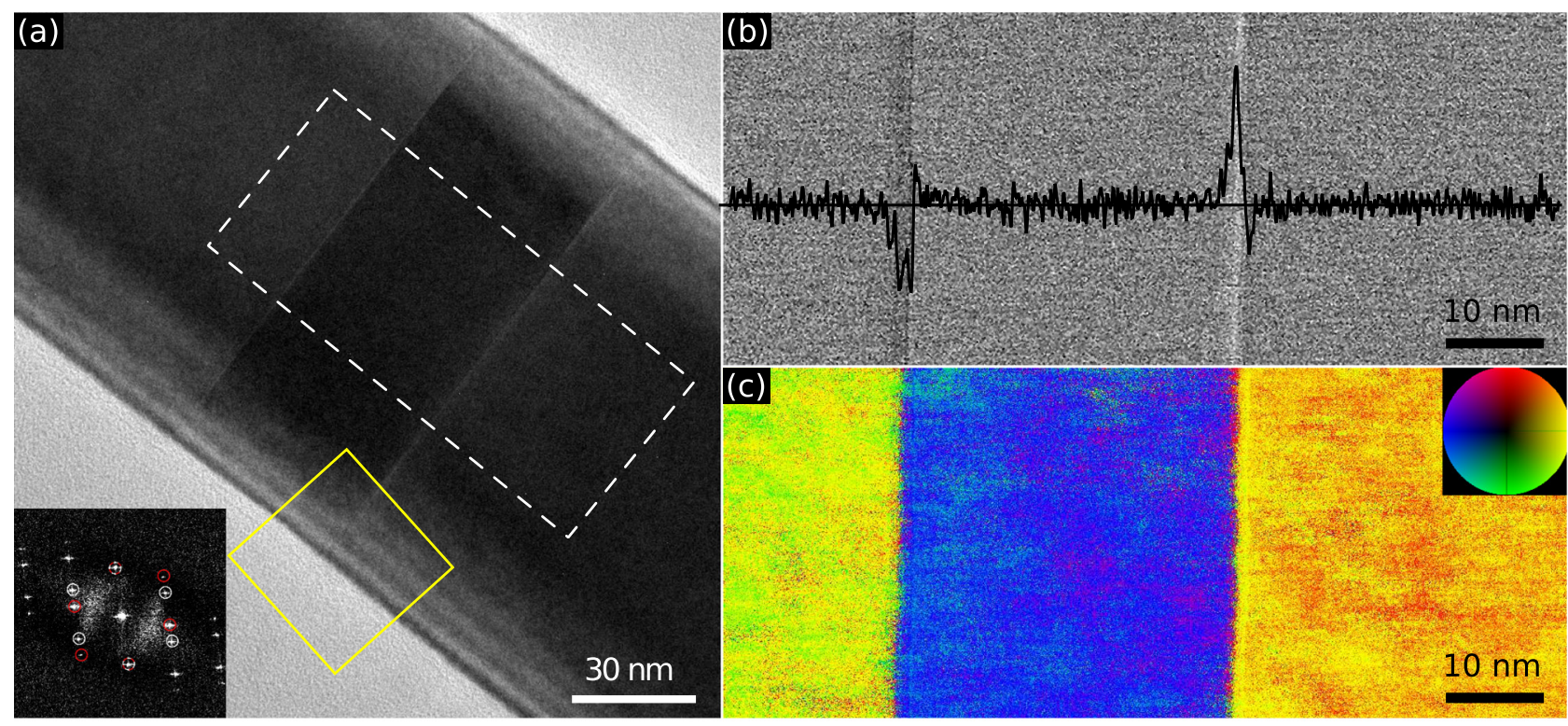

FIG. 3. The double twin defect structure used for polarization quantification: (a) TEM overview of the structure. The inset shows the fast Fourier transform of a high resolution image taken from the square marked region with the typical spot pattern of twinned $\mathrm{ZB}$ structure. The dashed frame indicates the location where images (b) and (c) were taken. (b) Charge distribution map with superimposed line scan profile. (c) Mapping of the electric field: field direction is colorcoded according to the inset color wheel, color brightness corresponds to field strength.

between electron probe profile and sample structure which further complicates their interpretation. Also it turns out that the four-quadrant detector setup used in this experiment does not allow for quantitative measurements on crystal lattice length scales. ${ }^{19}$ For that reason, a direct quantitative access to the spontaneous polarization in WZ is not possible with the given setup. However, there is no such limitation for fields that can be considered homogeneous over areas larger than the beam diameter.

Thus, for a quantification of $P_{\mathrm{sp}}$ a feature is needed where such a homogeneous field over several nanometers length can be expected. The feature that is most well-defined in this context is the twin defect in the $\mathrm{ZB}$ structure with a stacking order of $A B C B A$, i.e., the stacking order is simply reversed. The $B C B$ part can be considered as the smallest possible WZ segment embedded on both sides into ZB material, where the latter is free of polarization. If two such twins are located at a distance which is small compared to the NW diameter, this arrangement can be treated as a plate capacitor where the sheet charges at the twin defects act as capacitor plates filled with a dielectric of susceptibility ${ }^{20} \varepsilon_{\mathrm{GaAs}}=13.17$.

An arrangement as described above was present in the sample as depicted in Fig. 3(a). For quantitative measurements, it is important that the entry and exit surfaces of the sample are parallel ${ }^{15}$ which is ensured by the fact that the NW is lying on one of the six $\{11 \overline{2} 0\}$ side facets and is oriented in the same zone axis. As GaAs NWs are known to grow in very regular hexagonal shape ${ }^{21-24}$ the transversed sample thickness $t$ can easily be determined by geometrical considerations from the observable width $w$ of the NW as $t=\frac{\sqrt{3}}{2} w=(111 \pm 5) \mathrm{nm}$. This thickness can be considered constant as long as the measurement is constricted to the region where the NW has plane-parallel surfaces as indicated by the dashed frame in Fig. 3(a).

The charge distribution map as well as the electric field map (Figs. 3(b) and 3(c)) clearly demonstrate the capacitor- like configuration of this structure: in the former a bright line at one and a dark line at the other interface indicate positive and negative sheet charges at those interfaces, while in the latter the electric field between the interfaces (blue) points opposite to the field on their outside (yellow). Figure 4 depicts the strength $|\vec{E}|$ of the electric field calculated according to Eq. (1) along a line through the center of the image area depicted in Figs. 3(b) and 3(c). As only the height of the step at the twin boundaries is of relevance, we subtracted a signal offset such that the average field outside the double twin structure is zero. The height of the step was determined by taking the average of the curve between the vertical dotted lines in Fig. 4 resulting in a value of $22.9 \mathrm{MV} / \mathrm{m}$ as marked in the curve with a blue dashed line. For both, the region inside and outside the double twin defect, the standard deviations, which are $0.7 \mathrm{MV} / \mathrm{m}$ and $0.9 \mathrm{MV} / \mathrm{m}$, respectively, are indicated by dotted lines. Together with the error

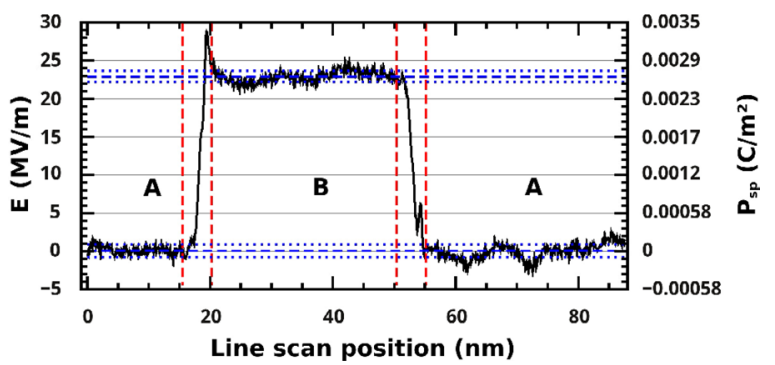

FIG. 4. Electric field strength along the line scan indicated in Fig. 3(b) but taken from Fig. 3(c). As only the step height is of importance, the curve was set such that the outside (regions marked "A") of the double twin structure has a mean value of zero to facilitate interpretation. The blue dashed and dotted lines represent the averages and standard deviations inside (B) and outside (A) of the double twin structure. The step edges between the vertical dashed lines were excluded as they contain measurement artifacts. On the right vertical axis the spontaneous polarization according to $P_{\mathrm{sp}}=\varepsilon \varepsilon_{0} E$ is displayed. 
margin from the thickness determination and the measurement setup itself this sums up to an electric field value of $(23 \pm 5) \mathrm{MV} / \mathrm{m}$. In terms of spontaneous polarization, this results in $P_{\mathrm{sp}}=(2.7 \pm 0.6) \times 10^{-3} \mathrm{C} / \mathrm{m}^{2}$ which agrees fairly well with the $(2 \ldots 3) \times 10^{-3} \mathrm{C} / \mathrm{m}^{2}$ predicted by Belabbes et al. ${ }^{9}$

Using DPC in a STEM, we could show unambiguously that there is a spontaneous polarization within the WZ phase of GaAs NWs and measure its strength. It turns out to be significant although an order of magnitude smaller than in the III-Nitrides. The resulting internal fields will have implications on the electronic band structure via the QCSE and therefore on the optical properties of NWs, supporting a recent interpretation of luminescence spectra. ${ }^{13}$

We gratefully acknowledge financial support by the German Research Foundation (DFG) via Grants SFB 689 "Spin Phenomena in Reduced Dimensions" and "Focused Researcher's Group: PolarCoN (FOR 957)."

${ }^{1}$ V. G. Dubrovskii and N. V. Sibirev, Phys. Rev. B 77, 035414 (2008).

${ }^{2}$ S. Noor Mohammad, J. Appl. Phys. 110, 084310 (2011).

${ }^{3}$ M. Rosini and R. Magri, ACS Nano 4, 6021 (2010).

${ }^{4}$ J. P. Boulanger and R. R. LaPierre, J. Cryst. Growth 332, 21 (2011).

${ }^{5}$ Y. A. Du, S. Sakong, and P. Kratzer, Phys. Rev. B 87, 075308 (2013).

${ }^{6}$ C.-Y. Yeh, Z. W. Lu, S. Froyen, and A. Zunger, Phys. Rev. B 46, 10086 (1992).

${ }^{7}$ R. Gurwitz, A. Tavor, L. Karpeles, I. Shalish, W. Yi, G. Seryogin, and V. Narayanamurti, Appl. Phys. Lett. 100, 191602 (2012).

${ }^{8}$ M. I. McMahon and R. J. Nelmes, Phys. Rev. Lett. 95, 215505 (2005).
${ }^{9}$ A. Belabbes, J. Furthmüller, and F. Bechstedt, Phys. Rev. B 87, 035305 (2013).

${ }^{10}$ J. Lähnemann, O. Brandt, U. Jahn, C. Pfüller, C. Roder, P. Dogan, F. Grosse, A. Belabbes, F. Bechstedt, A. Trampert, and L. Geelhaar, Phys. Rev. B 86, 081302 (2012).

${ }^{11}$ S. C. Jain, M. Willander, J. Narayan, and R. Van Overstraeten, J. Appl. Phys. 87, 965 (2000).

${ }^{12}$ D. A. B. Miller, D. S. Chemla, T. C. Damen, A. C. Gossard, W. Wiegmann, T. H. Wood, and C. A. Burrus, Phys. Rev. Lett. 53, 2173 (1984).

${ }^{13}$ U. Jahn, J. Lähnemann, C. Pfüller, O. Brandt, S. Breuer, B. Jenichen, M. Ramsteiner, L. Geelhaar, and H. Riechert, Phys. Rev. B 85, 045323 (2012).

${ }^{14}$ J. Chapman, P. Batson, E. Waddell, and R. Ferrier, Ultramicroscopy 3, 203 (1978).

${ }^{15}$ M. Lohr, R. Schregle, M. Jetter, C. Wächter, T. Wunderer, F. Scholz, and J. Zweck, Ultramicroscopy 117, 7 (2012).

${ }^{16}$ N. Shibata, S. D. Findlay, Y. Kohno, H. Sawada, Y. Kondo, and Y. Ikuhara, Nature Phys. 8, 611 (2012).

${ }^{17}$ The linear proportionality between $E \times t$ and signal intensity is based on the $\sin (x) \approx x$ approximation, which holds true as the deflection angle in our case is in the mrad range or below.

${ }^{18}$ M. Soda, A. Rudolph, D. Schuh, J. Zweck, D. Bougeard, and E. Reiger, Phys. Rev. B 85, 245450 (2012).

${ }^{19} \mathrm{~K}$. Müller, "On the measurement of atomic scale electric fields," private communication (2014).

${ }^{20}$ D. D. Nolte, in Properties of Gallium Arsenide, 3rd ed., edited by M. R. Brozel and G. E. Stillmann (INSPEC, 1995), Vol. 16, p. 201.

${ }^{21}$ A. Rudolph, M. Soda, M. Kiessling, T. Wojtowicz, D. Schuh, W. Wegscheider, J. Zweck, C. Back, and E. Reiger, Nano Lett. 9, 3860 (2009).

${ }^{22}$ A. Fontcuberta i Morral, D. Spirkoska, J. Arbiol, M. Heigoldt, J. R. Morante, and G. Abstreiter, Small 4, 899 (2008).

${ }^{23}$ D. Spirkoska, G. Abstreiter, and A. Fontcuberta i Morral, Semicond. Sci. Technol. 24, 113001 (2009).

${ }^{24}$ M. J. Tambe, L. F. Allard, and S. Gradečak, J. Phys.: Conf. Ser. 209, 012033 (2010). 